\title{
Performance of a 1.2 THz Frequency Tripler using a GaAs Frameless Membrane Monolithic Circuit
}

\author{
Alain Maestrini ${ }^{1}$, Jean Bruston ${ }^{2}$, David Pukala ${ }^{1}$, Suzanne Martin ${ }^{1} \&$ Imran Mehdi $^{1}$ \\ ${ }^{1}$ Jet Propulsion Laboratory, MS 168-314, 4800 Oak Grove Drive, Pasadena, CA 91109 \\ ${ }^{2}$ Now at ESA - ESTEC, Noordwijk, NL
}

\begin{abstract}
The first ever planar Schottky diode multiplier working over a THz will be presented in this paper. A tunerless $1.2 \mathrm{THz}$ waveguide frequency tripler has been designed, fabricated and tested. The frequency multiplier consists of a 3 micron-thick GaAs frameless-membrane monolithic circuit, mounted in a split waveguide-block, which includes a built-in Picket-Potter horn. The 1.2 THz membrane tripler is driven by a $400 \mathrm{GHz}$ solid-state chain composed of HEMT based power amplifiers followed by two tunerless planar diode frequency doublers. At room temperature, output power up to 80 microwatts was measured at $1126 \mathrm{GHz}$ with a peak-efficiency of $0.9 \%$ and a $3 \mathrm{~dB}$ bandwidth of about $3.5 \%$. The output power of the multiplier chain increased dramatically with a decrease of the ambient temperature up to 195 microwatts was measured at $120 \mathrm{~K}$. When further cooled to $50 \mathrm{~K}$ the chain delivers power levels as high as 250 microwatts. To the best of our knowledge, this is the first demonstration of a fully planar multiplier chain at these frequencies, along with performance that supercedes current state-of-the-art performance of whisker-contacted sources.
\end{abstract}

\section{INTRODUCTION}

Several astrophysical and Earth observation space missions, planned for the near future, will require submillimeter-wave heterodyne radiometers for spectral line observations. Hot Electron Bolometers and SIS based junctions are planned to be utilized as ultra sensitive detectors in the 1.2 $\mathrm{THz}$ to $2.4 \mathrm{THz}$ range on those space missions. The required Local Oscillator (LO) sources to pump the mixers are critical to the successful implementation of those missions and will be the focus of this paper.

Multiplied sources in the $\mathrm{THz}$ regime are essential for compact space instruments since there are no other easily viable alternatives. While the mixer and detector technology has progressed extremely well over the last decade or so, multiplier sources continue to be the bottleneck towards successful instrumentation. To date there have been only a handful of demonstrations of solid-state sources above $1 \mathrm{THz}$, all utilizing whisker contacted Schottky diodes, which require tedious assembly processes without the ability of multiple diode design. In addition, the per- formance in terms of bandwidth and efficiency is difficult to reproduce when the active device has to be replaced or re-contacted, which continues to be a source of concern when used in space programs. The highest frequency result reported to date is a $1395 \mathrm{GHz}$ tripler [1] that produces $17 \mu \mathrm{W}$ of power at an input power of $7 \mathrm{~mW}$ (from a Carcinotron). On the other hand, planar Schottky varactor diodes allow much better reproducibility of performance. Considerable efficiency and output power have been demonstrated up to the $300 \mathrm{GHz}$ range, mostly based on the planar balanced doubler concept proposed and demonstrated by Neal Erickson [2], [3], [4]. Discrete planar devices continue to work well into the $300 \mathrm{GHz}$ range but, as the operating frequency is further increased, the limitations of the assembly process soon become formidable. At the Jet Propulsion Laboratory a concerted effort has been made to develop and demonstrate technology that makes the implementation relatively straightforward while allowing the design to be scaled into the $\mathrm{THz}$ range. Within this context, the present paper will discuss the implementation of a solid-state source to $1.2 \mathrm{THz}$ with allplanar diode multipliers. Special emphasis is placed on the final stage tripler $(1.2 \mathrm{THz})$ that utilizes several novel technologies.

\section{DESIGN AND FABRICATION PROCESS}

The 1.2 THz membrane tripler concept was described previously in [5], whereas the fabrication process of the chip was described with more detail in [6]. It consists of a splitblock waveguide tripler using two Schottky planar varactor diodes in anti-parallel configuration.

The waveguide block includes a $1.2 \mathrm{THz}$ Picket-Potter dual mode feed-horn that was machined in two symmetrical parts, using commercial and custom-made milling tools. The circuit is integrated on a three-micron thick frameless GaAs membrane. It is located between the input waveguide and the output waveguide, inside an $80 \times 80 \times 155$ micron channel. The $400 \mathrm{GHz}$ pump signal is coupled to the device by a 175 micron-long E-plane probe, 
whereas the output signal at $1.2 \mathrm{THz}$ is coupled to the output waveguide by a 35 micron-long E-plane probe (see Figure 1). A matching circuit of only two elements is used to reduce the RF losses and the physical dimensions of the chip while improving, respectively, the conversion efficiency and the mechanical ruggedness. Two one-micronthick gold beam-leads located on the side of the membrane support the chip above the bottom half of the channel. When the top and bottom halves of the block are assembled, the beam- leads insure a ground path.

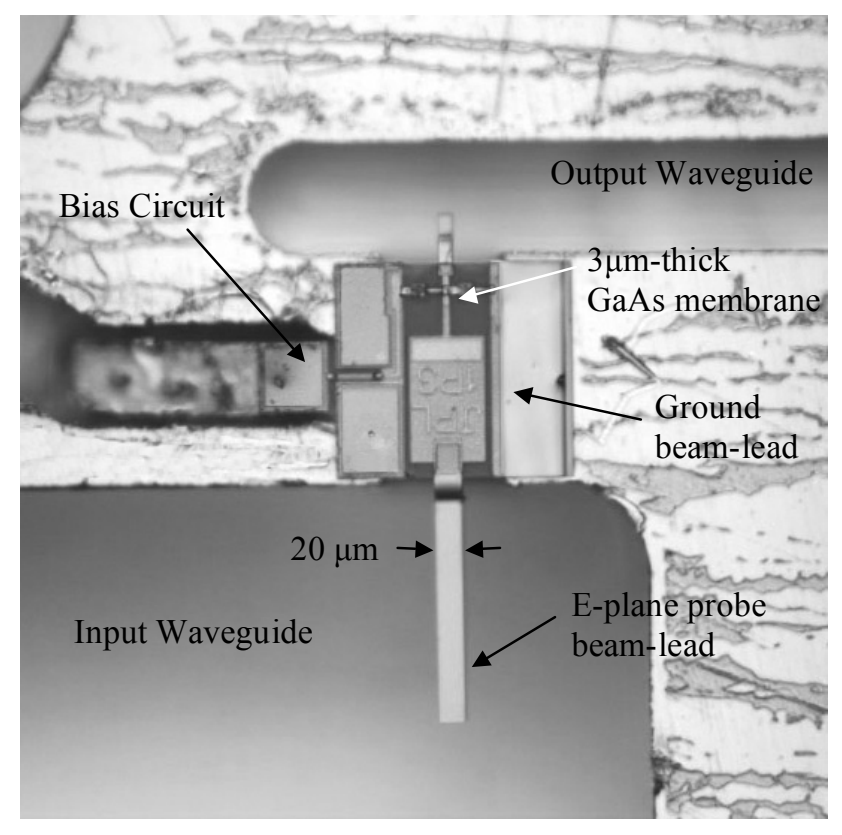

Fig.1. The frameless membrane circuit in the block. The size of the chip without the input and output probes is about $150 \times 160$ $\mu \mathrm{m}$.

As described in [5], the two-step design philosophy consists in determining, first, the parameters of the diode that can give the best conversion efficiency for a given frequency, input power and fabrication process, and then, to optimize the matching circuit. The optimization of the diode is made possible by the use of a non-linear model of the planar Schottky diodes fabricated at JPL [7].

In this design, $5 \mathrm{~mW}$ of pump power was assumed for each diode. The optimal anode size was found to be around $0.4 \times 1.0$ micron for an epitaxial doping of $5 \times 10^{17} \mathrm{~cm}^{-3}$. The circuit itself was constrained dramatically by the size and RF loss considerations. In order to achieve a good match at the input and output frequency with very few tuning elements, we chose to use E-plane input and output probes as part of the input and output matching circuit.
Two families of devices were designed and fabricated: one with an integrated circuit that allows the biasing of the diodes, and one with no bias circuit. Given the relatively low level of pump power at $400 \mathrm{GHz}$, the simulations show that the optimum bias is fairly close to zero volt and that a bias-less circuit should work. Moreover, the biasless approach offers a number of distinct advantages: the devices are easier to fabricate and much easier to mount in the waveguide block. On the other hand, with no bias capability, it is impossible to make any in-block device diagnostics. In addition, since the current in the diodes cannot be monitored, it is impossible to estimate the input coupling.

In spite of the very thin membrane, it was not difficult to mount the unbiased devices in the waveguide blocks. The GaAs membrane and the large beam leads are fairly robust features that allow one to handle and place the devices as desired.

\section{ROOM TEMPERATURE RF MEASUREMENTS}

A solid-state source at $400 \mathrm{GHz}$, described in [8], was used to pump the $1.2 \mathrm{THz}$ membrane tripler. Note that the only non solid-state element of the chain is the BWO fundamental source (used for convenience and labavailability), and that it could be easily replaced by an active multiplier or a medium power Gunn source. Power amplifiers at $100 \mathrm{GHz}$ [9] were incorporated in the chain to increase the pump power of the $200 \mathrm{GHz}$ doubler (designed by Neal Erickson at U-Mass). This multiplier is a discrete planar diode balanced doubler that gives $40 \mathrm{~mW}$ at $188 \mathrm{GHz}$ with an efficiency of $20 \%$. The second stage multiplier at $400 \mathrm{GHz}$ uses a "substrate-less" planar circuit [10]. All the multipliers are tunerless. At room temperature, this chain delivers $8 \mathrm{~mW}$ at $375 \mathrm{GHz}$.

The output power at $1.2 \mathrm{THz}$ tripler was measured using a photo-acoustic detector (from Thomas Keating LTD), considered as a reliable power meter at sub-millimeter wavelengths. Unfortunately the sensitivity of this detector is not high enough to easily measure signals below a few microwatts. In addition, when the RF power produced by the multiplier chain is below 50 microwatts, bias optimization is hardly possible, due to the long integration time necessary to get an acceptable signal-to-noise ratio.

In order to get a calibrated measurement at $1.2 \mathrm{THz}$, we combined in the same test setup a Thomas Keating detector and a liquid-helium cooled silicon bolometer. The setup is shown in Figure 2. Thanks to the high sensitivity of the bolometer, the multiplier chain output power was easily maximized by optimizing the biases of the $200 \mathrm{GHz}$ and $400 \mathrm{GHz}$ multipliers. By increasing the integration time, the power was measured with the Thomas Keating detector. A noise floor of about $\pm 0.5 \mu \mathrm{W}$ was reached af- 
ter 10-15 minutes of integration. The RF losses of the cryostat vacuum window were calibrated using this setup. Due to the short distance between the detector and the multiplier (about $30 \mathrm{~mm}$ ), the losses introduced by the water vapor were small, except at frequencies close to the absorption lines $(1109.6,1113.3,1146.6,1153.3,1155.1$, $1158.3,1162.9,1168.3,1172.5$ and $1190.8 \mathrm{GHz})$. The losses introduced by the atmosphere were not taken into account for the experiments presented in this paper.

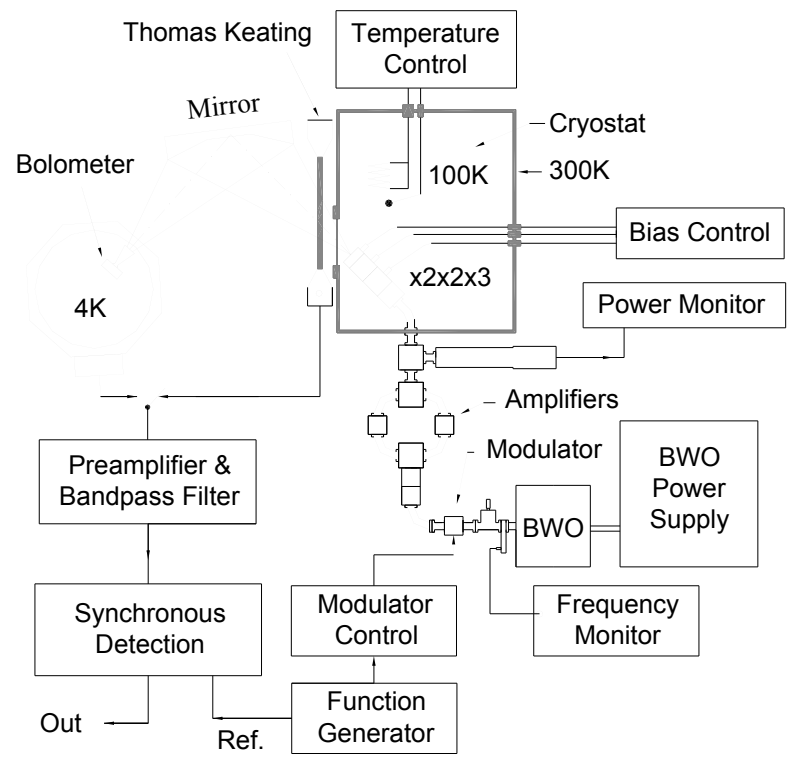

Fig.2. Block diagram of the cryogenic test bench.

When using a bias-less circuit with two 0.4 by 0.9 micron anodes, signals up to 70 microwatts at $297 \mathrm{~K}$ were measured, with an efficiency of $0.9 \%$ for the $1.2 \mathrm{THz}$ tripler. The bandwidth was found to be $3.5 \%$. The measured performance is shown in Figure 3. It is important to note that the performance of the $1.2 \mathrm{THz}$ tripler depends strongly on the performance of the lower frequency stages. With input power at $400 \mathrm{GHz}$ below $4 \mathrm{~mW}$, the $1.2 \mathrm{THz}$ tripler does not deliver more than 12 microwatts. This suggests that the intrinsic bandwidth of the $1.2 \mathrm{THz}$ tripler could be much wider. Unfortunately, we did not have a $400 \mathrm{GHz}$ chain powerful enough to measure the performance of the 1.2 THz tripler over a wider range of frequencies.

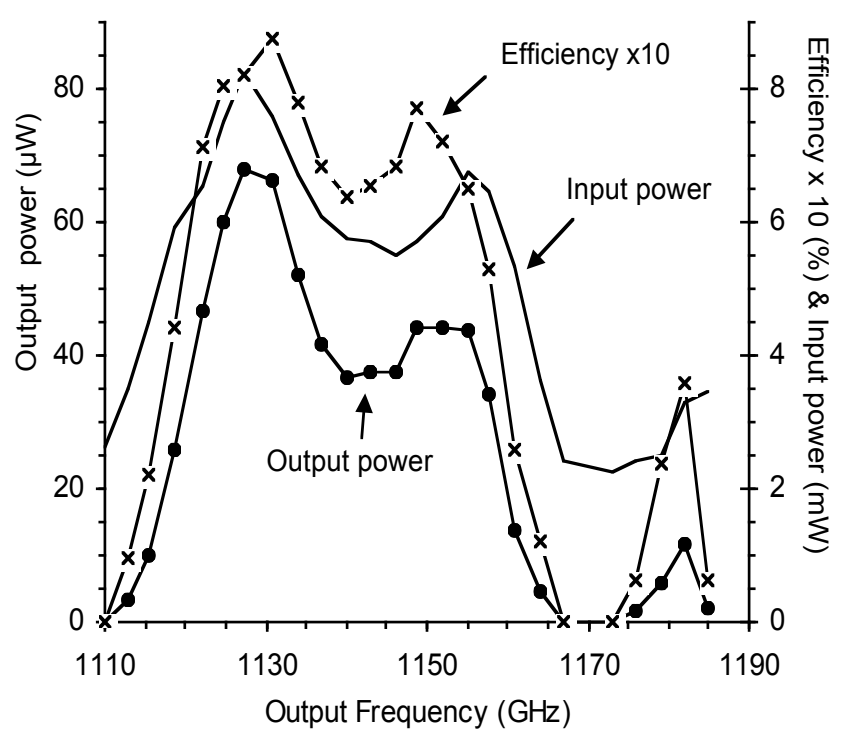

Fig.3. Output power of the $1.2 \mathrm{THz}$ multiplier chain measured at 297K. Bias voltages on the $200 \mathrm{GHz}$ and $400 \mathrm{GHz}$ multipliers are optimized for each frequency.

\section{CRYOGENIC RF MEASUREMENTS}

Decreasing the operating temperature has shown to drastically improve varactor performance especially when a number of multiplier stages are cascaded together [8], [11]. As apparent in Figure 2, the multiplier stages are mounted inside of a temperature-controlled cryostat. In this particular setup the amplifiers are outside the cryostat although there is the possibility of cooling them to further increase the input power.

Figure 4 shows the output power of the $1.2 \mathrm{THz}$ chain at temperatures ranging from $297 \mathrm{~K}$ to $50 \mathrm{~K}$. Note that the data collected in Figure 4 were obtained with the same $1.2 \mathrm{THz}$ tripler and the same $400 \mathrm{GHz}$ doubler but with a different $200 \mathrm{GHz}$ doubler. This new $200 \mathrm{GHz}$ multiplier provided slightly more power than the previous one. Consequently, we measured, at room temperature, $80 \mathrm{mi}-$ crowatts at $1126 \mathrm{GHz}$ instead of 70 microwatts as obtained in Figure 3. An improvement of $3.5 \mathrm{~dB}$ in the output power was observed when the chain was cooled from $300 \mathrm{~K}$ to $150 \mathrm{~K}(175 \mu \mathrm{W})$, an increase of $4.6 \mathrm{~dB}$ was observed when cooled from $300 \mathrm{~K}$ to $120 \mathrm{~K}(195 \mu \mathrm{W})$, and an increase of $5 \mathrm{~dB}$ was measured when cooled from $300 \mathrm{~K}$ to $50 \mathrm{~K}(250 \mu \mathrm{W})$. These power levels are the highest reported to date for a solid state local oscillator chain working above $1 \mathrm{THz}$. 


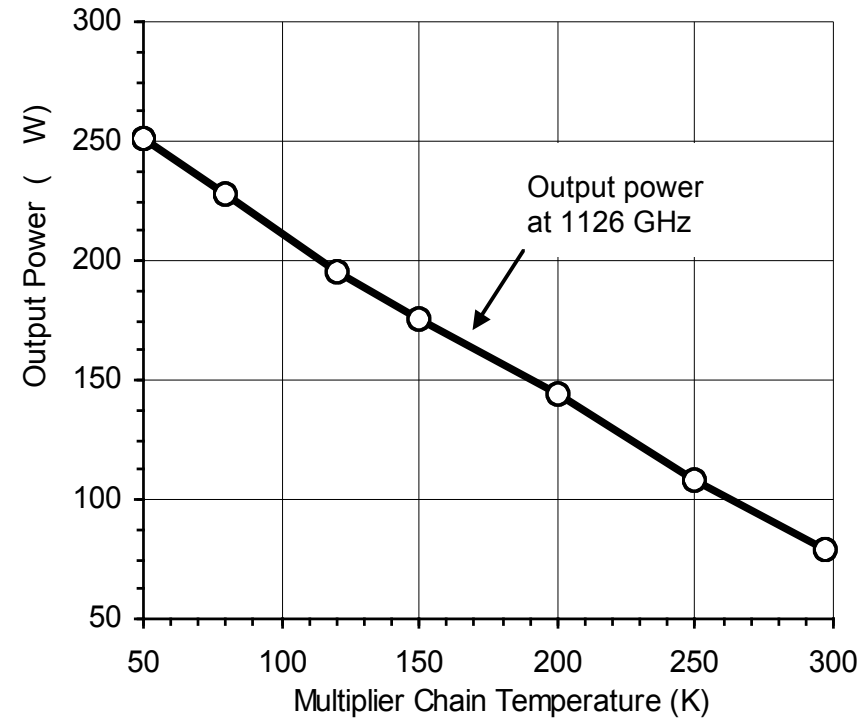

Fig.4. Output power of the $1.2 \mathrm{THz}$ multiplier chain at $1126 \mathrm{GHz}$ measured from $297 \mathrm{~K}$ to $50 \mathrm{~K}$. Bias voltages on the $200 \mathrm{GHz}$ and $400 \mathrm{GHz}$ multipliers are optimized for each temperature (optimized biases are in fact non temperature dependent).

\section{CONCLUSION}

A first ever demonstration of a planar multiplier working over a $\mathrm{THz}$ has been presented. The complete source to $1200 \mathrm{GHz}$ is based on two balanced planar Schottky diode varactors followed by a balanced planar tripler. At room temperature the measured output power of $80 \mu \mathrm{W}$ was observed. Upon cooling the output power increased dramatically reaching a level of $250 \mu \mathrm{W}$ at $50 \mathrm{~K}$. It is worth noting that the technology developed for this LO chain is scaleable, and that work is in progress to further optimize this chain along with increasing the operating frequency.

\section{ACKNOWLEDGEMENTS}

Technical support from L. Samoska, Todd Gaier, B. Nakamura, and A. Fung are greatly appreciated. The authors wish to thank Peter Bruneau and the JPL Space Instruments Shop for the superb fabrication of the waveguide blocks. Technical discussions with P. Siegel, J. Pearson, N. Erickson (U-Mass) and P. Zimmermann (RPG) are also acknowledged. The research described in this publication was carried out at the Jet Propulsion Laboratory, Califor- nia Institute of Technology, under a contract with the National Aeronautics and Space Administration.

\section{REFERENCES}

[1] P. Zimmermann, "Solid-State Oscillators for the THzRange", proceeding of the $8^{\text {th }}$ International Conference on Terahertz Electronics, Darmstadt, 28-29 September 2000.

[2] N. Erickson, "High efficiency submillimeter frequency multipliers", IEEE MTT-S Digest, pp.1301-1304, 1990.

[3] N. Erickson, "THz Frequency Multipliers for FIRST and SOFIA", Proceedings of $2^{\text {nd }}$ ESA Workshop on Millimetre Wave Technology and Applications: Antennas, Circuits and systems, pp. 423-428, May 1998.

[4] N. Erickson, R. P. Smith, S. C. Martin, B. Nakamura, and I. Mehdi, "High Efficiency MMIC Frequency Triplers for Millimeter and Submillimeter Wavelengths," IEEE MTT Symposium, Boston, June 2000.

[5] J. Bruston, E. Schlecht, A. Maestrini, F. Maiwald, S.C. Martin, R.P. Smith, and I. Mehdi, P.H. Siegel \& J. Pearson " Development of $200 \mathrm{GHz}$ to $2.7 \mathrm{THz}$ Multiplier Chains for Submillimeter-wave Heterodyne Receivers", proceeding of the SPIE-International Symposium on Astronomical Telescope and Instrumentation, 27-31 March 2000.

[6] J. Bruston, S. Martin, A. Maestrini, E. Schlecht, P. Smith, and I. Mehdi, "The Frameless Membrane: a Novel Technology for THz Circuits", proceeding of the $11^{\text {th }}$ International Space Terahertz Technology Symposium, Ann Arbor, June 2000.

[7] J. Bruston, R.P. Smith, S.C. Martin, A. Pease and P.H. Siegel, "Progress Toward the Realization of MMIC Technology at Submillimeter Wavelengths: A Frequency Multiplier to $320 \mathrm{GHz}$," Proc. IEEE Intl. Microwave Symp. Dig., Baltimore, MD, June 1998, pp. 399-402.

[8] A. Maestrini, D. Pukala, F. Maiwald, E. Schlecht, G. Chattopadhyay, and I. Mehdi, "Cryogenic operation of GaAs based multiplier chains to $400 \mathrm{GHz}$ ", proceeding of the $8^{\text {th }}$ International Conference on Terahertz Electronics, Darmstadt.

[9] L. Samoska, T. Gaier, A. Peralta, S. Weinreb, J. Bruston, I. Mehdi, Y.C. Chen, H.H. Liao, M. Nishimoto, R. Lai, H. Wang, Y.C. Leong, "MMIC Power Amplifiers as Local Oscillators for FIRST," Proc. SPIE UV, Optical, and IR Space Telescopes and Instruments, SPIE 4013, Munich, Germany, March 2000.

[10] E. Schlecht, J. Bruston, A. Maestrini, S. Martin, D. Pukala, R. Tsang, A. Fung, R. P. Smith ${ }^{\dagger}$, I. Mehdi, "200 and $400 \mathrm{GHz}$ Schottky Diode Multipliers Fabricated with Integrated Air-Dielectric 'Substrateless' Circuitry", $11^{\text {th }}$ International Symposium on Space Terahertz Technology, Ann Arbor, Michigan, May1-3, 2000.

[11] J. T. Louhi, A. V. Raisanen, and N. R. Erickson, "Cooled Schottky Varactor Frequency Multipliers at Submillimeter Wavelengths," IEEE Trans. MTT, vol. 41, pp. 565-571, 1993. 